\title{
Optimal power control in CDMA over Markov fading channels
}

\author{
Ngoc Thanh Bui \\ ARC Special Research Centre for \\ Ultra-Broadband Information Networks \\ Department of Electrical \& Electronic \\ Engineering \\ University of Melbourne \\ Parkville, Victoria 3010 Australia \\ e-mail: ntbeee.mu.oz.au
}

\begin{abstract}
This paper obtains a new centralized stochastic power control algorithm for a DS/CDMA single cell uplink environment where the channel fading process is modeled by a stationary finite state Markov chain. Constrained stochastic approximation methods can be used to solve the stochastic optimization problem.
\end{abstract}

\section{INTRODUCTION}

In this paper, we propose a new centralized stochastic power control algorithm for a single cell DS/CDMA uplink environment where the effect of channel fading is taken into account. We formulate the power control problem as a constrained optimization (minimization) where the objective function is the sum of all mobile transmit powers and the constraints represent the target average (with respect to the fading statistics) signal to interference ratio (ASIR) for all mobile users. Each user's uplink channel is modeled as a stationary finite state Markov chain, although this algorithm can be extended to other statistical models. Ideally speaking, one should work with outage probability constraints, but often these outage probabilities cannot be expressed in closed form or may even be discontinuous functions of the optimization variables. If all the parameters of the channel fading statistics are known, and there is no feedback channel noise between the base station and the mobile users, one can use iterative deterministic constrained optimization methods based on penalty multiplier type algorithms as in [1]. In practice, however, these parameters are only estimated by channel estimation algorithms and there is feedback channel noise and therefore one needs to use constrained stochastic approximation methods [2] to solve the stochastic optimization problem. As the constraints are not always convex, one can only obtain local minima (under certain conditions holding) and the stochastic approximation methods converge to the Karush-Kuhn-Tucker points of the corresponding deterministic optimization methods with probability one. We also show that the stochastic power control algorithm can be combined with a linear minimum mean square error multiuser detector (LMMSE MUD) using a coupled optimization technique.

\section{DS/CDMA SYSTEM MODEL AND PROBLEM FORMULATION}

Consider a synchronous binary DS/CDMA communication system with $K$ active users transmitting through an additive Gaussian white noise channel, where the channel gains are modeled as independent stationary finite state Markov chains. In our simulations, we take each channel to have a "good" state and a "bad" state, although these results can be generalized to any number of states. The output $S I R_{k}$ of user $k$, which for any receiver vector $c_{k}$ and a channel gain combination $\left(h_{1 m}, \ldots, h_{K m}\right)$ can be written as

$$
S I R_{k m}=\frac{p_{k} h_{k m}\left(c_{k}^{T} s_{k}\right)^{2}}{\sum_{j=1, j \neq k}^{K} p_{j} h_{j m}\left(c_{k}^{T} s_{j}\right)^{2}+\sigma^{2} c_{k}^{T} c_{k}}
$$

\author{
Subhrakanti Dey \\ ARC Special Research Centre for \\ Ultra-Broadband Information Networks \\ Department of Electrical \& Electronic \\ Engineering \\ University of Melbourne \\ Parkville, Victoria 3010 Australia \\ e-mail: sdeydee.mu.oz.au
}

where $p_{k}, s_{k}$ are the transmitted power and the normalized signature sequence of the $k$-th user and $\sigma^{2}$ is the noise variance. Obviously, $m \in\left\{1,2, \ldots, 2^{K}\right\}$. The optimization problem is formulated in the following way:

$$
\begin{aligned}
\operatorname{minimize} f(p) & =\sum_{k=1}^{K} p_{k} \\
\text { s.t. } \quad g_{k}(p)=\gamma_{k}-\overline{S I R_{k}} & \leq 0 \quad \text { for } k=1,2, \ldots, K . \\
\overline{S I R_{k}} & =\sum_{m=1}^{M} S I R_{k m} \pi(m)
\end{aligned}
$$

where vector $p=\left[p_{1}, p_{2} \ldots p_{K}\right]^{T}, \gamma_{k}$ is the target SIR of user $k$, $M=2^{K}$ and $\pi(m)$ is the stationary probability of the combined Markov channel being in the $m$-th state..

\section{SiMUlation STUDiES}

This section provides some simulation results to demonstrate the convergence of our stochastic power control algorithm with ASIR constraints over a fading channel modeled as a first-order, 2-state Markov chain with 4 users (with target ASIR as $6 \mathrm{~dB}$ ) where we combine our algorithm with an average LMMSE MUD receiver. The only source of noise comes from a white Gaussian noisy feedback channel. The following graph shows the convergence of the stochastic power control algorithm.

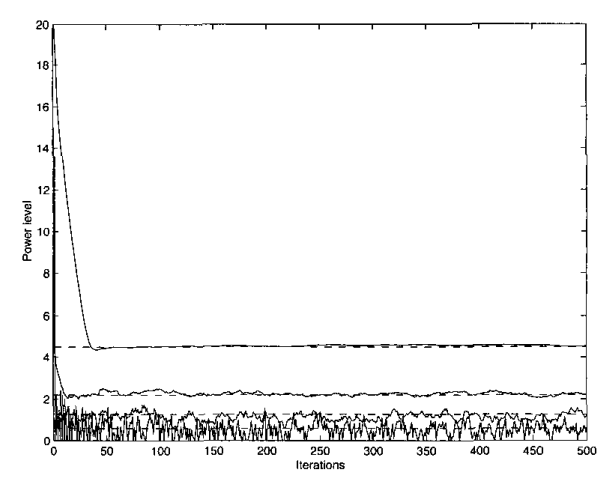

(a) Average LMMSE case

\section{REFERENCES}

[1] D. P. Bertsekas, Nonlinear programming Second edition, Athena Scientific, Massachusetts, 1999.

[2] H. J. Kushner. Stochastic approximation methods for constrained and unconstrained systems, Springer-Verlag, New York, 1978. 